\title{
Digital twin of mechatronic drive based on the optimal control model of BLDC motor
}

\author{
Sergei Trefilov* \\ Department of Mechatronic Systems, Kalashnikov Izhevsk State Technical University, 426069, \\ Studencheskaya str, 7, Izhevsk, Russia
}

\begin{abstract}
This paper presents a digital twin of a mechatronic drive based on a brushless DC (BLDC) motor model based on a nonlinear discrete optimal control model. Some parameters of a BLDC motor, such as resistance and inductance of windings, magnetic flux, viscous friction coefficient in bearings, angular velocity and electromagnetic moment, can change due to both degradation of structural elements and external forces. Simulation by a complete enumeration of the values of the parameters of the mechatronic device with a certain step will make it possible to adapt the program of the control device to changing operating conditions according to the criterion of minimizing the control energy by changing the parameters of the state matrices and control of the digital twin. As a result, the accuracy of the movement of the mechatronic device along the given trajectory will increase due to the greater correspondence of the control parameters to the real object.
\end{abstract}

\section{Introduction}

Currently, in connection with the development in industry of complex and capital-intensive production systems, accidents on which can lead to significant losses due to expensive repairs, and the significant development of data transmission facilities, computer technology and storage devices for large amounts of data, the concept of digital twins has appeared. [15]. Behind this concept lies an approach based on forecasting methods, for example, the residual resource of elements of complex systems [1-5]. Methods and mathematical models for such forecasting, based on the methods of mathematical statistics, were widely developed in the 20th century. Now this approach, based on recording a large amount of measurement data in the database, makes it possible to form training samples of conditionally "suitable", that is, those capable of fulfilling a functional purpose with a high probability, and conditionally "unusable elements of systems that can lead to an accident. the entire system soon and must be replaced immediately. Another approach involves modeling the parameters of the control algorithm in order to adapt it to changing operating conditions both due to material degradation and environmental changes.

The purpose of the digital twin is determined in accordance with [1-5] as a software model, with the help of which it is possible to determine the degree of conformity of the

\footnotetext{
* Corresponding author: trefilov376908@gmail.com
} 
control model incorporated in the control program of the mechatronic device and the real parameters of the drive. If during the operation of the mechatronic device the parameters change due to the degradation of the nodes, then during control the errors of the output parameter (angular speed of the drive) will increase. To ensure the specified accuracy of the angular speed of the drive, an algorithm for adapting the control program is proposed based on modeling the state and control matrices [6-18].

This paper presents a mathematical model of a digital twin for a BLDC motor, as an element of a production line, and optimally controlled using a nonlinear algorithm. Modeling the operating conditions from the model will allow the controller to adapt to changing operating conditions.

\section{Mathematical model of mechatronic drive}

It is known that the optimal control in the state space is represented by the following model in discrete form, when the matrix $\mathbf{A}_{k}, \mathbf{B}_{k}, \mathbf{C}_{k}$ are constant at times $k, k=0,1,2,3, \ldots$ [11].

$$
\begin{gathered}
\frac{\mathbf{x}_{k+1}-\mathbf{x}_{k}}{T}=\mathbf{A}_{k} \mathbf{x}_{k}+\mathbf{B}_{k} \mathbf{u}_{k}, \\
\mathbf{y}_{\boldsymbol{k}}=\mathbf{C}_{\boldsymbol{k}} \mathbf{x}_{\boldsymbol{k}},
\end{gathered}
$$

where $\mathbf{x}_{k}, \mathbf{x}_{k+1}$ are state vectors on the trajectory of motion,

$\mathbf{A}_{k}, \mathbf{B}_{k}, \mathbf{C}_{k}$ - matrices of state, control and measurement, $\mathbf{y}_{k}$ is the measured output state of the system,

$T$ is the sampling time.

Let us represent (1) in the classical form

$$
\mathbf{x}_{k+1}=\widetilde{\mathbf{A}}_{k} \mathbf{x}_{k}+\widetilde{\mathbf{B}}_{k} \mathbf{u}_{k}
$$

$$
\text { where } \widetilde{\mathbf{A}}_{k}=T \mathbf{A}+\mathbf{E}, \quad \widetilde{\mathbf{B}}_{k}=T \mathbf{B}_{k} \text {. }
$$

We find solution (2) as a solution to the matrix equation, i.e. the state score will be calculated as:

$$
\hat{\mathbf{x}}_{k}=\mathbf{C}_{k}^{-1} \mathbf{y}_{k}
$$

The optimal solution (3) with the view (4) is found in the form of [12-17]:

$$
\mathbf{u}_{k}=-\mathbf{G}_{k}^{-1} \mathbf{B}_{k}^{T} \mathbf{K}_{k} \hat{\mathbf{x}}_{k}
$$

where $\mathrm{K}_{k}$ is the Cauchy matrix, which can be found by solving the Riccati equation $[16,17]$ :

$$
-\dot{\mathbf{K}}=\mathbf{Q}+\mathbf{A}^{T} \mathbf{K}+\mathbf{K}^{T} \mathbf{A}-\mathbf{K}^{T} \mathbf{B} \mathbf{G}^{-1} \mathbf{B}^{T} \mathbf{K}
$$

where $\mathrm{Q}$ and $\mathrm{G}$ are positive arbitrarily defined matrices. The matrices $\mathrm{Q}$ and $\mathrm{G}$ are chosen positive definite, since affect only the scale of the solution. We will select these matrices by selection or modeling [16,17].

The quality criterion that minimizes the energy of control and displacement, in this case, is a quadratic form: 


$$
\mathbf{I}=\frac{1}{2} \int_{t_{0}}^{t_{f}}\left(\mathbf{x}^{T} \mathbf{Q} \mathbf{x}+\mathbf{u}^{T} \mathbf{G u}\right) d t, \mathbf{Q} \geq 0, \mathbf{G}>0
$$

The control process assumes adjusting the matrix $\mathbf{A}_{k}$ at each control step. In the initial serviceable state of the mechatronic device, only one element will change in this matrix. This element contains a variable load moment, which is calculated according to the data of the information-measuring system in terms of the BLDC motor shaft as

$$
\begin{gathered}
M(k)=\varepsilon(k) J, \\
M(k)=\frac{\omega(k+1)-\omega(k)}{T} J, \\
\boldsymbol{\omega}(\boldsymbol{k}+\mathbf{1})=\frac{\theta(k+1)-\theta(k)}{T},
\end{gathered}
$$

where $\varepsilon(k)$ is the angular acceleration reduced to the BLDC motor shaft, which is necessary to return to the trajectory set during control;

$J$ is the moment of inertia of the mechatronic device reduced to the electric drive shaft.

Let the accuracy of the trajectory execution by the mechatronic device be given as $\delta_{\max }$, determined by the tolerances for the manufacture of the components of the mechatronic device and the permissible environmental parameters of the functioning of this mechatronic device. The control device at the moment of loss of controllability according to the criterion of an increase in the trajectory errors above the given $\delta_{\max }$ or the impossibility of performing control due to the exceeding of the passport values of the parameters of the electric motor starts the algorithm for modeling the state and control matrices on the digital twin.

The program for modeling the state and control matrices of the digital twin can be placed on a high-performance external processor that receives data from the memory, into which the control unit of the mechatronic device places the current state and control matrices at regular intervals while moving along a certain trajectory segment. On the other hand, if the frequency of control measurements is large, and the processor power of the control device is sufficient, then simulation of the state and control matrices can be implemented in the background on the device itself.

The following algorithm is proposed for modeling the state and control matrices on a digital twin to ensure the minimum control energy for a given accuracy of the angular speed of the drive.

1. Initial data for modeling:

- the designated section of the trajectory of movement in the form of a vector $\mathrm{s}_{k}$ consisting of elements $s(k), k=1,2, \ldots, K$;

- state matrix $\mathrm{A}_{k}\left(\omega_{k} \mid \mathbf{s}(k)\right)=\left[a_{i j}(k)\right], k=\overline{\mathbf{1}, K}, i, j=\overline{\mathbf{1}, N}$;

- the control matrix $\mathbf{B}_{k}\left(\omega_{k} \mid \mathbf{s}(k)=\left[b_{p q}(k)\right], k=\overline{1, K}, p=\overline{1, N}, q=\overline{1, M}, M \leq N\right.$;

- $\boldsymbol{n}_{\boldsymbol{i} j}, \boldsymbol{n}_{p q}= \pm \mathbf{0}, \mathbf{1}, 2, \ldots, \boldsymbol{i j}=\overline{\mathbf{1}, \boldsymbol{N} * \boldsymbol{N}}, \boldsymbol{p q}=\overline{\mathbf{1}, \boldsymbol{N} * \boldsymbol{M}}$ are indices of enumeration of model options.

2. We will change the parameters of the control and state matrices for all indices $i, j, p, q$ of matrices A, B and the indices of enumeration of degradation options $n_{i j}, n_{p q}$ for all points of the trajectory

$$
\begin{gathered}
\mathbf{s}\left(k \mid \boldsymbol{n}_{i j}, \boldsymbol{n}_{p q}\right), \boldsymbol{k}=\mathbf{1}, 2, \ldots, \boldsymbol{n}_{i j}, \boldsymbol{n}_{p q}= \pm \mathbf{0}, 1,2, \ldots . \\
\mathbf{A}_{k}\left(i, j, n_{i j}\right)=\left[\boldsymbol{a}_{i j}(k) \pm \boldsymbol{n}_{i j} \Delta \boldsymbol{a}_{i j}, \boldsymbol{n}_{i j}=\mathbf{0}, \mathbf{1}, 2, \ldots\right], \\
\mathbf{B}_{k}\left(\boldsymbol{p}, \boldsymbol{q}, \boldsymbol{n}_{i j}, \boldsymbol{n}_{p q}\right)=\left[\boldsymbol{b}_{p q}(k) \pm \boldsymbol{n}_{p q} \Delta \mathbf{b}_{p q}, \boldsymbol{n}_{p q}=\mathbf{0}, 1,2, \ldots\right] .
\end{gathered}
$$


$\Delta \boldsymbol{a}_{i j}$ и $\Delta \mathbf{b}_{p q}$ are the increments for matrix elements that determine the accuracy of modeling the state and control matrices $\mathbf{A}, \mathbf{B}$.

3. Let us calculate the standard deviation of the control voltage over all components of the control vector $\mathrm{u}_{k}$ with the components at each step $\boldsymbol{u}_{\boldsymbol{k}}(\boldsymbol{n}), \boldsymbol{n}=\overline{\mathbf{1}, \boldsymbol{N}}$, determined according to (5):

$$
\sigma^{2}\left(u_{K}, s_{K} \mid i, j, p, q, i j, p q\right)=\frac{1}{K} \sum_{k=1}^{K}\left(u_{k}^{2}(1)+u_{k}^{2}(2)+\cdots+u_{k}^{2}(N)\right) .
$$

This value, up to a constant coefficient, can be interpreted as the control energy $E$ on the trajectory section $\boldsymbol{s}(\boldsymbol{k}), \boldsymbol{k}=\mathbf{1}, 2, \ldots, K$;

4. We define the combination of control and state matrices corresponding to the minimum standard deviation as:

$$
\begin{aligned}
& A_{k} \mid \min \left(\sigma^{2}\left(u_{K}, s_{K} \mid i, j, p, q, i j, p q\right)\right)=\left[a_{i j}(k)\right], \\
& B_{k} \mid \min \left(\sigma^{2}\left(u_{K}, s_{K} \mid i, j, p, q, i j, p q\right)\right)=\left[b_{p q}(k)\right],
\end{aligned}
$$

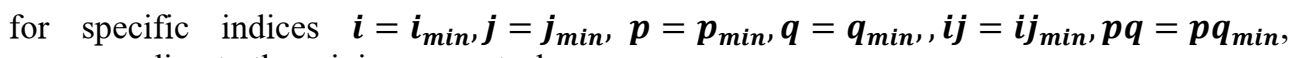
corresponding to the minimum control energy.

5. The calculated trajectory during simulation will be equal to

$$
\hat{\mathbf{s}}(\boldsymbol{k})=\hat{\mathbf{x}}_{k}=\boldsymbol{A}_{k-1}\left(\boldsymbol{i}_{\min }, \boldsymbol{j}_{\min }\right) \widehat{\boldsymbol{x}}_{k-1}+\boldsymbol{B}_{k-1}\left(\boldsymbol{p}_{\min }, \boldsymbol{q}_{\min }\right) \boldsymbol{u}_{k-1}
$$

Constant elements of control and state matrices do not change during brute-force simulation. A dynamic model of the BLDC motor was developed in a rotating coordinate system $(\mathrm{d}, \mathrm{q})$, oriented along the magnetic axis of the rotor. Based on the second Kirchhoff's law, an equation has been compiled that describes the electrical part of the BLDC motor in the d, $\mathrm{q}$ coordinate system rotating at the rotor speed $\omega$ :

$$
\overline{u_{s}}=\overline{\boldsymbol{l}_{s}} R_{s}+L_{s} \frac{d \bar{\iota}_{s}}{d t}+j \omega L_{s} \overline{\boldsymbol{l}_{s}}+j \omega \overline{\Phi_{0}}
$$

where $\overline{\boldsymbol{u}_{\boldsymbol{s}}}=\boldsymbol{u}_{\boldsymbol{s} \boldsymbol{d}}-\boldsymbol{j} \boldsymbol{u}_{\boldsymbol{s q}}$ is the resulting voltage vector on the stator winding;

$\boldsymbol{R}_{\boldsymbol{s}}, \boldsymbol{L}_{\boldsymbol{s}}$ are active resistance and total inductance of the stator phase;

$\overline{\Phi_{0}}=\Phi_{0 d}+j \Phi_{0 q}$ is the magnetic flow vector of the BLDC motor;

$\boldsymbol{j} \boldsymbol{\omega} \boldsymbol{L}_{\boldsymbol{s}} \overline{\boldsymbol{l}_{\boldsymbol{s}}}$ is voltage drop caused by the stator magnetic flux;

$\boldsymbol{j} \boldsymbol{\omega} \Phi_{0}$ is voltage drop caused by the main magnetic flux of the BLDC motor, which is created by the rotor.

Let us represent a model of a mechatronic drive based on a BLDC motor in the form $(1,2)$, taking $\mathbf{C}=\mathbf{E}$ :

$$
\begin{gathered}
\mathbf{x}(k+1)=\left[\begin{array}{c}
i_{d}(k+1) \\
i_{q}(k+1) \\
\omega(k+1) \\
\theta(k+1)
\end{array}\right]=\left[\begin{array}{cccc}
1-T \frac{R}{L} & T p \omega(k) & 0 & 0 \\
-T p \omega & 1-T \frac{R}{L} & -T \frac{K_{m}}{L} & 0 \\
0 & T \frac{K_{m}}{J} & 1-T \frac{F}{J}-T \frac{M(k)}{J \omega(k)} & 0 \\
0 & 0 & T & 1
\end{array}\right]\left[\begin{array}{c}
i_{d}(k) \\
i_{q}(k) \\
\omega(k) \\
\theta(k)
\end{array}\right]+\left[\begin{array}{cc}
\frac{T}{L} & 0 \\
0 & \frac{T}{L} \\
0 & 0 \\
0 & 0
\end{array}\right]\left[\begin{array}{l}
u_{d}(k) \\
u_{q}(k)
\end{array}\right] \\
{\left[\begin{array}{c}
\hat{i}_{d}(k) \\
\hat{i_{q}}(k) \\
\hat{\omega}(k) \\
\hat{\theta}(k)
\end{array}\right]=\left[\begin{array}{cccc}
1 & 0 & 0 & 0 \\
0 & 1 & 0 & 0 \\
0 & 0 & 1 & 0 \\
0 & 0 & 0 & 1
\end{array}\right]\left[\begin{array}{c}
i_{d}(k) \\
i_{q}(k) \\
\omega(k) \\
\theta(k)
\end{array}\right]+\left[\begin{array}{l}
\xi_{i d} \\
\xi_{i q} \\
\xi_{\omega} \\
\xi_{\theta}
\end{array}\right]}
\end{gathered}
$$


where $i_{d}(k), i_{q}(k), \omega(k), \theta(k)$ are the measured value of currents, angular velocity and displacement;

$\omega(k+1)$ is the planned value of the angular speed;

$\theta(k+1)$ is the planned value of the angular position of the motor shaft;

$T$ is sampling interval, time between $k+1$ and $k$ samples;

$R$ is the active resistance of the stator winding of the BLDC motor;

$L_{q}, L_{d}$ are inductance of the BLDC motor stator along the q and $\mathrm{d}$ axes;

$p$ is the number of pairs of poles of the BLDC motor;

$\psi$ is the magnetic flux induced by permanent magnets in the stator winding;

$F$ is coefficient of viscous friction in the BLDC motor;

$J$ is moment of inertia;

$M(k)$ is the electromagnetic moment of the BLDC motor;

$i_{d}(k+1), i_{q}(k+1), \omega(k+1), \theta(k+1)$ are projection of the stator current on the d-axis, projection of the stator current on the q axis, the angular velocity, angular displacement of the BLDC motor at time $k+1$;

$u_{q}, u_{d}$ are stator voltage projections on the $\mathrm{q}$ and $\mathrm{d}$ axes;

$\left[\begin{array}{llll}\xi_{i d} & \xi_{i q} & \xi_{\omega} & \xi_{\theta}\end{array}\right]^{T}$ is measurement error vector;

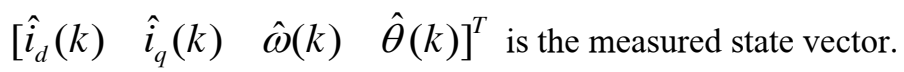

The novelty of the discrete BLDC motor model is the presence of a variable load moment in the state matrix at each step of the simulation, calculated according to the required moment for movement along a given trajectory, based on the measured trajectory. Then the state matrix of the BLDC motor model will be equal to

$$
\mathbf{A}=\left[\begin{array}{cccc}
1-T \frac{R}{L} & T p \omega(k) & 0 & 0 \\
-T p \omega & 1-T \frac{R}{L} & -T \frac{K_{m}}{L} & 0 \\
0 & T \frac{K_{m}}{J} & 1-T \frac{F}{J}-T \frac{M(k)}{J \omega(k)} & 0 \\
0 & 0 & T & 1
\end{array}\right] .
$$

Control matrix is:

$$
\mathbf{B}=\left[\begin{array}{cc}
\frac{T}{L} & 0 \\
0 & \frac{T}{L} \\
0 & 0 \\
0 & 0
\end{array}\right]
$$

Variable in the process of operation and simulated components $R, L$ for the state matrix (15) and $L$ for the control matrix (16) of the BLDC motor model.

\section{BLDC motor simulation results}

Let us calculate the control and state matrices for the BLDC motor JK42BLS01. The parameters of the BLDC motor JK42BLS01 are shown in table 1. 
Table 1. Technical characteristics of BLDC motor JK42BLS01

\begin{tabular}{|l|l|}
\hline BLDC motor technical characteristics & $\begin{array}{l}\text { Parameter } \\
\text { value }\end{array}$ \\
\hline Weight, $\mathrm{kg}$ & 0.3 \\
\hline Length, mm & 41 \\
\hline Diameter, mm & 20 \\
\hline Supply voltage, V & 24 \\
\hline Winding resistance, Ohm & 19 \\
\hline Winding inductance, H & 0.0018 \\
\hline Idling speed, rpm & 7400 \\
\hline No-load current, A & 0.05 \\
\hline Rated current, A & 1.8 \\
\hline Rated moment, N·m & 0.0625 \\
\hline Moment of inertia, $\mathrm{kg} \cdot \mathrm{m} 2$ & 0.0000024 \\
\hline Constant torque coefficient, N/A & 0.039 \\
\hline Constant back-EMF coefficient, V/rpm & 0.0041 \\
\hline Power, W & 26 \\
\hline Rated angular speed, rpm & 4000 \\
\hline Number of poles & 8 \\
\hline Magnetic flux,Wb & 0.001 \\
\hline
\end{tabular}

The state matrix of the BLDC motor JK42BLS01 model will be equal to

$$
\mathbf{A}=\left[\begin{array}{cccc}
1-T * 1056 & T * 8 * \omega(k) & 0 & 0 \\
-T * 8 * \omega & 1-T * 1056 & -T * 0.444 & 0 \\
0 & T * 33.333 & 1-T * 41.667-T \frac{M(k)}{0.0000024 * \omega(k)} & 0 \\
0 & 0 & T & 1
\end{array}\right]
$$

The control matrix of the BLDC motor JK42BLS01 model will be equal to

$$
\mathbf{B}=\left[\begin{array}{cc}
555.55 & 0 \\
0 & 555.55 \\
0 & 0 \\
0 & 0
\end{array}\right]
$$

Let's set the measurement matrix $\mathbf{C}$ in the form of a unit matrix, we get:

$$
\left[\begin{array}{c}
\widehat{\iota_{d}} \\
\widehat{\iota_{q}} \\
\widehat{\omega} \\
\widehat{\theta}
\end{array}\right]=\left[\begin{array}{l}
i_{d} \\
i_{q} \\
\omega \\
\theta
\end{array}\right]+\left[\begin{array}{l}
\xi_{I_{d}} \\
\xi_{I_{q}} \\
\xi_{\omega} \\
\xi_{\theta}
\end{array}\right],
$$

where $\left[\begin{array}{llll}\xi_{i d} & \xi_{i q} & \xi_{\omega} & \xi_{\theta}\end{array}\right]^{T}$ is the vector of measurement errors that can be simulated by a pseudo-random number generator. In this experiment, the vector of control errors is taken equal to zero.

The BLDC motor JK42BLS01 was simulated in the MatLab program. Time $T=0.001 \mathrm{sec}$. 
Figure 1 shows the projections of the voltage vector $U d, U q$ of the BLDC motor stator, calculated on the basis of the Riccati equation in the MatLab program.

Figure 2 shows the projections of the control currents id and iq.

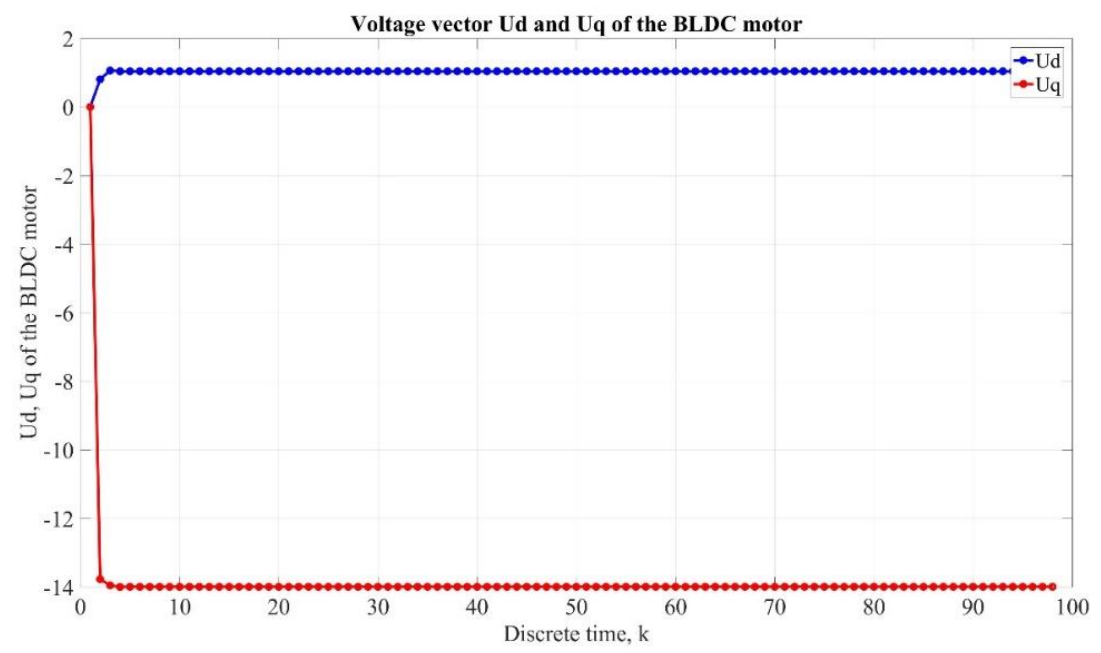

Fig. 1. Projections of the voltage vector Ud, Uq of the stator, calculated on the basis of the Riccati equation.

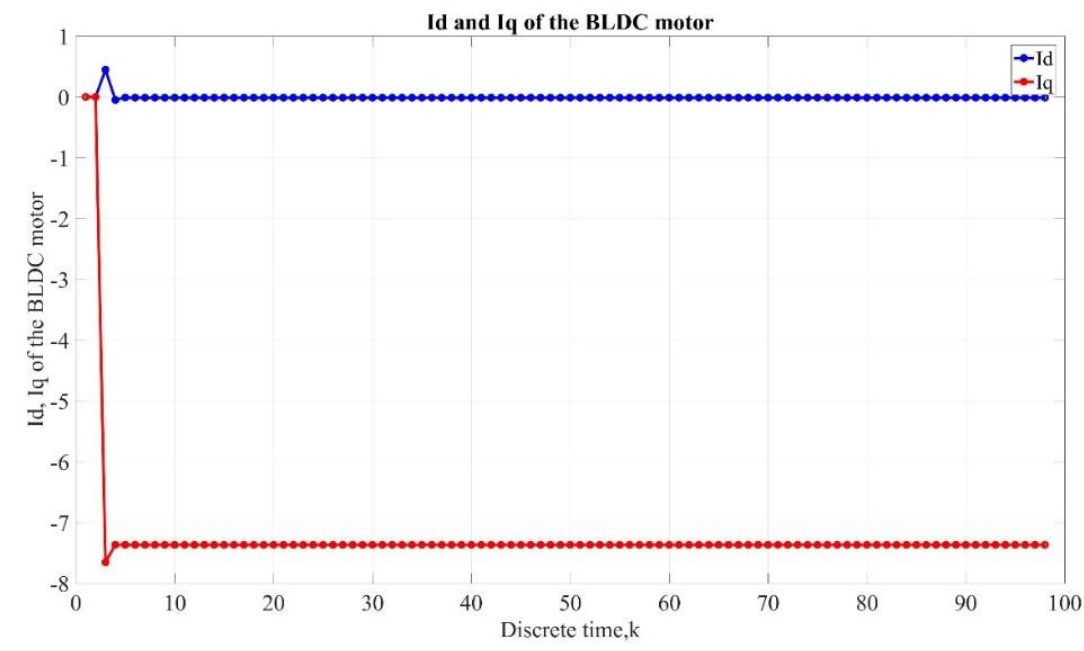

Fig. 2. Projections of the electric current vector Id, Iq of the stator.

The selection of the elements of the state and control matrices according to the algorithm as a result of modeling and the recording of these values into the control algorithm at the control object will ensure the adaptation of the control program to changing operating conditions.

\section{Conclusion}

This paper presents a digital twin of a mechatronic drive based on a BLDC motor model based on a nonlinear discrete and optimal control model. Simulation on a digital twin by a 
complete enumeration of discrete values of the BLDC motor parameters with a search for elements of control and state matrices according to the criterion of the minimum control energy will make it possible to introduce a correction into the control program for the mechatronic device control device. The minimization of the control energy according to the proposed algorithm provides:

1. Optimal control of BLDC motor according to the criterion of minimum control energy.

2. Adaptation of the control algorithm to changing operating conditions.

3. Maintaining the dynamic range of control voltages at close to the original values of the voltage.

4. Increasing the accuracy of the reproduction of the trajectory in comparison with the current accuracy.

The mechanism of digital twins, which implements the proposed adaptation algorithm, can be implemented both on a processor external to the mechatronic device and on the processor of the control device for this mechatronic device.

The reported study was funded by RFBR, project number 18-08-00772 A.

\section{References}

1. W. Michael, Grieves Digital Twin: Manufacturing Excellence through Virtual Factory Replication. LLC, 7 p. (2014)

2. D. Tarkhov, A. Vasilyev, Semi-empirical Neural Network Modeling and Digital Twins Development. London. Academic Press. 240 p. (2020)

3. R. Kharat, V. Bavane, S. Jadhao, R. Marode, Digital Twin: Manufacturing Excellence through Virtual Factory Replication. Global journal of engineering science and researches. 10 p. (2018)

4. A.G. Kravets, A.A. Bolshakov, M.V. Shcherbakov, Cyber-Physical Systems: Advances in Design \& Modelling. Springer. 340 p. (2020)

5. E.A. Lee, Cyberphysical systems: Design challenges. 11th IEEE International Symposium on Object and Component-Oriented Real-Time Distributed Computing (ISORC). Orlando. IEEE. pp. 363-369. (2020)

6. K. Sang-Hoon, Electric Motor Control DC, AC and BLDC Motors. Elsevier Inc., 426 p. (2017)

7. P. Stepanov, Yu. Nikitin, Diagnostics of Mechatronic Systems on the Basis of Neural Networks with High-Performance Data Collection. Mechatronics 2013: Recent Technological and Scientific Advances. Springer Intrenational Publishing Swizerland, 2014. 7-9.10.2013, Brno, Czech Republic. Pp. 433-440. (2013)

8. Yu. Turygin, P. Božek, I. Abramov, Yu. Nikitin, Reliability Determination and Diagnostics of a Mechatronic System. Advances in Science and Technology. Vol. 12, No. 2, June, pp. 274-290. (2018)

9. S. Trefilov, Y. Nikitin, Automatic warehouses with transport robots of increased reliability. ActaLogistica, vol. 5, no. 3. Pp. 19-23. (2018)

10. P. Bozek, A. Shchenyatsky, Yu. Turygin, Yu. Nikitin, Yu. Karavaev, Reverse validation of a programmed robot trajectory based on INS. 12th International Conference ELEKTRO 2018. IEEE, Mikulov, Czech Republic. 21-23 May 2018. Pp. 1-4. (2018)

11. S.A. Trefilov, Yu.R. Nikitin, Robot drives diagnostics by identifiability criterion based on state matrix. Instrumentation Engineering, Electronics and Telecommunications 2019 : Proceedings of the V International Forum (Izhevsk, Russia, November 20-22, 2019). Izhevsk. Publishing House of Kalashnikov ISTU. 123 p. Pp.105-114. (2019)

12. P. Eykhoff, System Identification: Parameter and State Estimation. Wiley-Interscience, New York. 555 p. (1974) 
13. P. Eykhoff, ed., Trends and progress in system identification. Oxford, England: Pergamon. 402 p. (1981)

14. D. Graupe, Identification of system. New York, R.E.Krieger Publishing Company. 302 p. (1976)

15. L. Ljung, System identification. Theory for the user. 2nd ed. PTR Prentice Hall, Upper Saddle River. 609 p. (1999)

16. A. Sage, J. Melsa, System identification. New York, Academic press. 221 p. (1999)

17. A. Sage, J. Melsa, Estimation Theory with Applications to Communications and Control. New York, McGraw-Hill. 496 p. (1971)

18. H. Luo, Plug-and-Play Monitoring and Performance Optimization for Industrial Automation Processes. Springer Fachmedien Wiesbaden GmbH. 158 p. (2017) 